\title{
NOAA-20 VIIRS Thermal Emissive Bands On-Orbit Performance
}

\author{
Yonghong Li ${ }^{\mathrm{a}}$, Xiaoxiong Xiong ${ }^{\mathrm{b}}$, Jeff McIntire ${ }^{\mathrm{a}}$, \\ Amit Angal ${ }^{\mathrm{a}}$, Sergey Gusev ${ }^{\mathrm{c}}$, and Kwofu Chiang ${ }^{\mathrm{a}}$ \\ a'Science Systems and Applications Inc., 10210 Greenbelt Road, Lanham, MD 20706, USA \\ bSciences and Exploration Directorate, NASA/GSFC, Greenbelt, MD 20771, USA \\ 'Global Science \& Technology, Inc., 7855 Walker Drive, Greenbelt, MD 20770, USA
}

\begin{abstract}
The VIIRS instrument onboard the NOAA-20 satellite (launched on November 18, 2017) started to collect Earth-view data after its nadir door opened on December 13, 2017. Seven of the VIIRS bands, I4-5 and M12-16 are thermal emissive bands (TEBs), covering a spectral range from 3.6 to $12.5 \mu \mathrm{m}$. They began collecting valid data after the cold focal plane assembly (CFPA) cooled down to its nominal operating temperature on January 6, 2018. This paper will present the performance of each TEB, including calibration coefficients, noise equivalent differential temperature (NEdT), on-orbit calibration coefficient estimates from scheduled onboard blackbody warm-up and cool-down (WUCD) data, as well as related telemetry temperatures. Several methods are tested and compared in the WUCD data analysis for estimating the calibration coefficients. Based on the preliminary results, the NEdT of each band is well below the design specification and very close to that of the VIIRS onboard the Suomi National Polar-orbiting Partnership (SNPP) satellite. The detector gains appear stable for bands on the short- and mid-wave infrared CFPA, whereas the detector gains have larger than expected degradation for bands on the long-wave infrared CFPA during the early mission. All TEB related telemetry temperatures are stable. The on-orbit performance of NOAA-20 VIIRS TEB is compared with VIIRS onboard the SNPP.
\end{abstract}

Keywords: Visible Infrared Imaging Radiometer Suite (VIIRS); thermal emissive bands (TEB); calibration; blackbody (BB)

\section{INTRODUCTION}

The second Visible Infrared Imaging Radiometer Suite (VIIRS) instrument is onboard the NOAA-20 (N20) satellite launched on November 18, 2017. Similar to the VIIRS onboard the Suomi National Polar-orbiting Partnership (SNPP) satellite, N20 VIIRS has seven thermal emissive bands (TEBs) I4-5, M12-16 with bands I4, M12-13 (3.5 4.2 $\mu \mathrm{m})$ on the short- and mid-wave infrared (SMIR) focal plane and bands I5, M14-16 (8.3 13.1 $\mu \mathrm{m})$ on the long-wave infrared (LWIR) focal plane. The temperatures of these focal planes are controlled at about $80.5 \mathrm{~K}$, and so are referred to as cold focal plane assemblies (CFPAs). Each imagery band (I-band) consists of 32 detectors in the track direction and has 6400 samples in the scan direction within a scan angle range of $\pm 56^{\circ}$ off nadir and a spatial resolution is $375 \mathrm{~m}$ at nadir. Within the same scan angle range, a moderate-resolution band (M-band) has 16 detectors aligned in the track direction and 3200 samples for each detector in the scan direction with a spatial resolution of $750 \mathrm{~m}$ at nadir ${ }^{1}$.

Similar to other sensors, the VIIRS TEBs use a blackbody (BB) as an onboard calibrator. The observation of the space view (SV) provides a zero reference which gives a thermal background and electronic offset. The BB temperature $\left(T_{b b}\right)$ is controlled at $292.6 \mathrm{~K}$ during normal operation and its emissivity is close to 1 by design (greater than $0.996^{2}$ ). The onorbit changes of the TEB calibration coefficients are assessed by periodically scheduled BB warm-up and cool-down (WUCD) cycles that provide detector responses over a range of temperatures from instrument ambient ( 266 K) to 315 $\mathrm{K}$.

The nadir door of the N20 VIIRS was opened on December 13, 2017 and its cryo-radiator door was opened on January 3 , 2018. Valid TEB data had been collected since the CFPA cooled down to its nominal operating temperature on January 6 , 2018. After the cryo-radiator door was opened, icing in the optical path on the LWIR dewar was believed to cause a 
rapid degradation of the gain in the LWIR bands. After a mid-mission outgas operation, performed on March 12, 2018, the gains in these LWIR bands returned to pre-icing levels and have subsequently remained stable.

This paper presents the on-orbit performance of N20 VIIRS TEBs from the aspect of related telemetries, calibration scale factor (known hereafter as the F-factor), noise equivalent differential temperature (NEdT), as well as the WUCD data analysis. Section 2 introduces the TEBs and their calibration algorithm. The short-term and long-term performance of the on-orbit telemetry, calibration coefficients, as well as NEdT are presented in Section 3. Section 4 discusses the WUCD data analysis and reports the on-orbit status of the estimated calibration coefficients.

\section{NOAA-20 VIIRS TEBS AND THEIR CALIBRATION ALGORITHM}

The VIIRS TEB design specifications are listed in Table 1, including center wavelengths, typical brightness temperatures $\left(\mathrm{T}_{\text {typ }}\right)$, and NEdT specification at $\mathrm{T}_{\text {typ. }}$. Band M13 has two gain modes, low gain (LG) and high gain (HG). The performance of M13 with high gain mode will be presented in the following discussion. The low gain mode is designed for the application of fire detection and it uses a different calibration approach from the regular calibration. For band M16, there are two sets of 16 detectors, known as M16A and M16B. The band M16 data is the time delay integration of M16A and M16B.

Table 1 VIIRS thermal emissive bands.

\begin{tabular}{|c|c|c|c|c|c|c|c|}
\hline Band & I4 & I5 & M12 & M13 & M14 & M15 & M16 \\
\hline Center Wavelength $(\mu \mathrm{m})$ & 3.74 & 11.45 & 3.70 & 4.05 & 8.55 & 10.76 & 12.01 \\
\hline $\mathbf{T}_{\text {typ }}(\mathbf{K})$ & 270 & 210 & 270 & $\begin{array}{l}300(\mathrm{HG}) \\
380(\mathrm{LG})\end{array}$ & 270 & 300 & 300 \\
\hline $\mathbf{N E d T}_{\text {spec }}$ at $\mathbf{T}_{\text {typ }}(\mathrm{K})$ & 2.500 & 1.500 & 0.396 & $\begin{array}{l}0.107(\mathrm{HG}) \\
0.423(\mathrm{LG})\end{array}$ & 0.091 & 0.070 & 0.072 \\
\hline
\end{tabular}

The TEB calibration converts the background corrected digital response (dn) of a detector to the corresponding radiance $(L)$ using a quadratic model ${ }^{1,2}$

$$
L_{\text {det }}=F \cdot \sum_{i=0}^{2} c_{i} \cdot d n^{i} .
$$

The coefficients, $c_{\mathrm{i}}(\mathrm{i}=0,1,2)$, in the model are known as calibration coefficients. $F$ is the scale factor (called F-factor) that is calculated on a scan-by-scan basis from observations of the on-orbit BB. For each scan, the BB radiance to a detector can be calculated by

$$
L_{\text {det } \_B B}=R V S_{B B} \cdot L_{B B}+L_{\text {background }},
$$

where RVS is response versus scan-angle. The radiance of the background and the $\mathrm{BB}$ are determined by

$$
\begin{gathered}
L_{\text {background }}=\left(R V S_{B B}-R V S_{S V}\right) \cdot \frac{\left[1-\rho_{r t a}\right] L\left(T_{r t a}\right)-L\left(T_{\text {ham }}\right)}{\rho_{\text {rta }}}, \\
L_{B B}=\varepsilon \cdot L\left(T_{B B}\right)+(1-\varepsilon) \cdot L_{\text {component }}, \\
L_{\text {component }}=w_{\text {rta }} \cdot L\left(T_{r t a}\right)+w_{\text {sh }} \cdot L\left(T_{\text {sh }}\right)+w_{\text {cav }} \cdot L\left(T_{\text {cav }}\right) .
\end{gathered}
$$

$T$ represents temperature and $L(\bullet)$ is the temperature-dependent radiance. $\varepsilon$ and $w$ are the BB emissivity and the weighting values $\left(w_{r t a}+w_{s h}+w_{c a v}=1\right)$, which are determined from the prelaunch data analysis. rta, sh, cav, and ham represent the rotating telescope assembly (RTA), the BB shield, the instrument cavity, and half angle mirror (HAM), respectively. The F-factor for each scan is then calculated by

$$
F=\frac{L_{d e t_{\_} B B}}{\sum_{i=0}^{2} c_{i} \cdot d n_{B B}^{i}} .
$$

The coefficients, RVS, and the temperature dependent radiance in Eqs. (1)-(6) are determined on a per-scan basis via the on-orbit telemetry measurements and the look-up-tables (LUTs). Currently, the TEB LUTs are characterized based on the prelaunch data analysis. 


\section{NOAA-20 VIIRS TEB ON-ORBIT PERFORMANCE}

\subsection{N20 VIIRS TEB short-term variation}

As introduced in the Section 2, the TEB performance mainly depends on the temperatures of the CFPAs and the BB. The TEB calibration also relates to the temperatures of the instrument components. So the temperature changes are closely monitored.
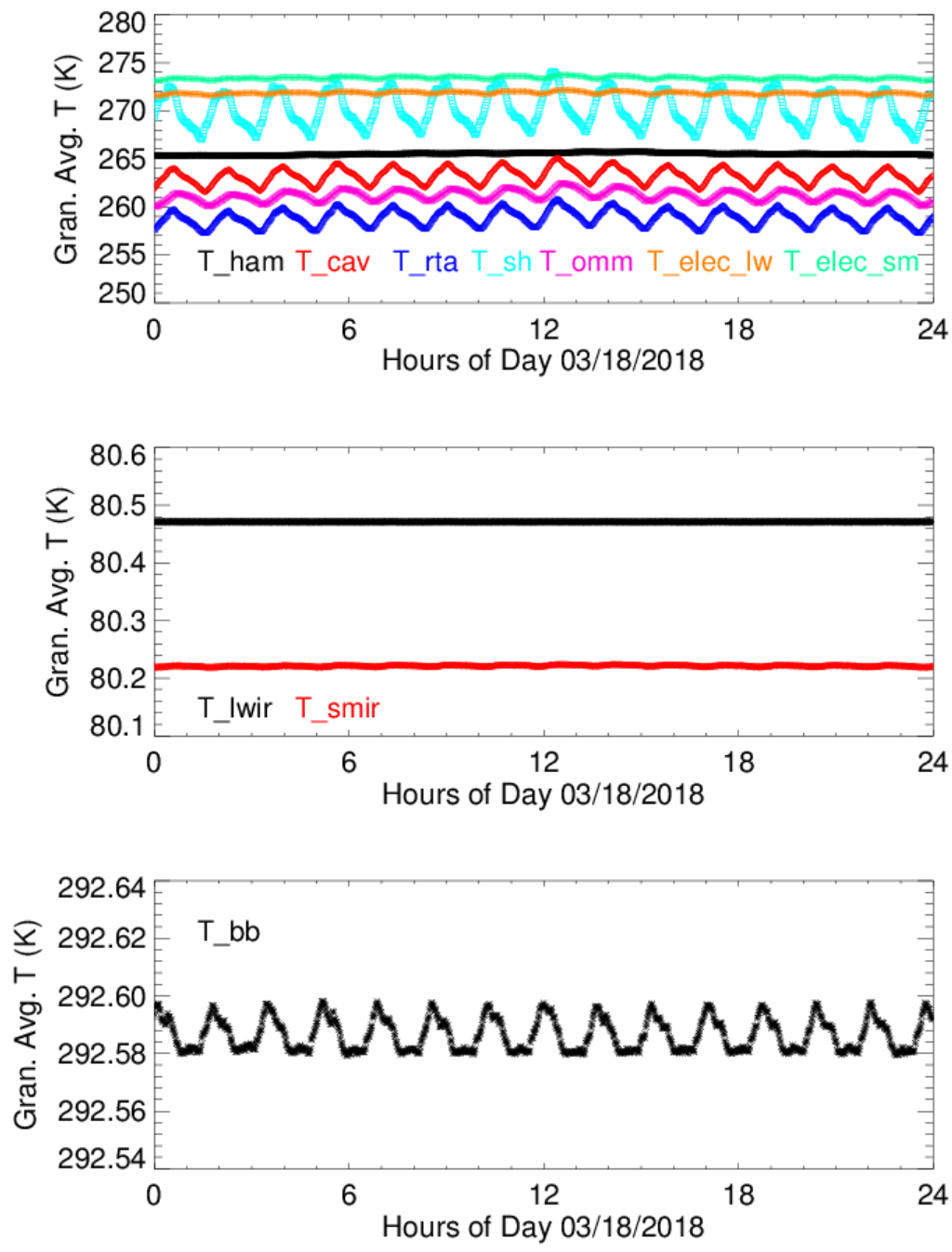

Figure 1. Daily trending of the granule-averaged temperatures on 03/18/2018.

Figure 1 illustrates daily trending plots of the granule averaged telemetry temperatures and the $\mathrm{T}_{\mathrm{bb}}$ on March 18, 2018. Orbital variations are seen in the temperatures of the cavity, the BB shield, the RTA, and the opto-mechanical module (OMM). The temperatures of the HAM and the electronics ( $T_{\text {elec_sm }}$ and $T_{\text {elec_ll }}$ ) have negligible orbital changes. The temperatures of the LWIR CFPA and the SMWIR CFPA are stable around $80.5 \mathrm{~K}$ and $80.2 \mathrm{~K}$, respectively. The granule averaged $\mathrm{T}_{\mathrm{bb}}$, the average of the six individual thermistor readings, also has orbital changes with higher temperature during daytime and relatively stable at nighttime. 

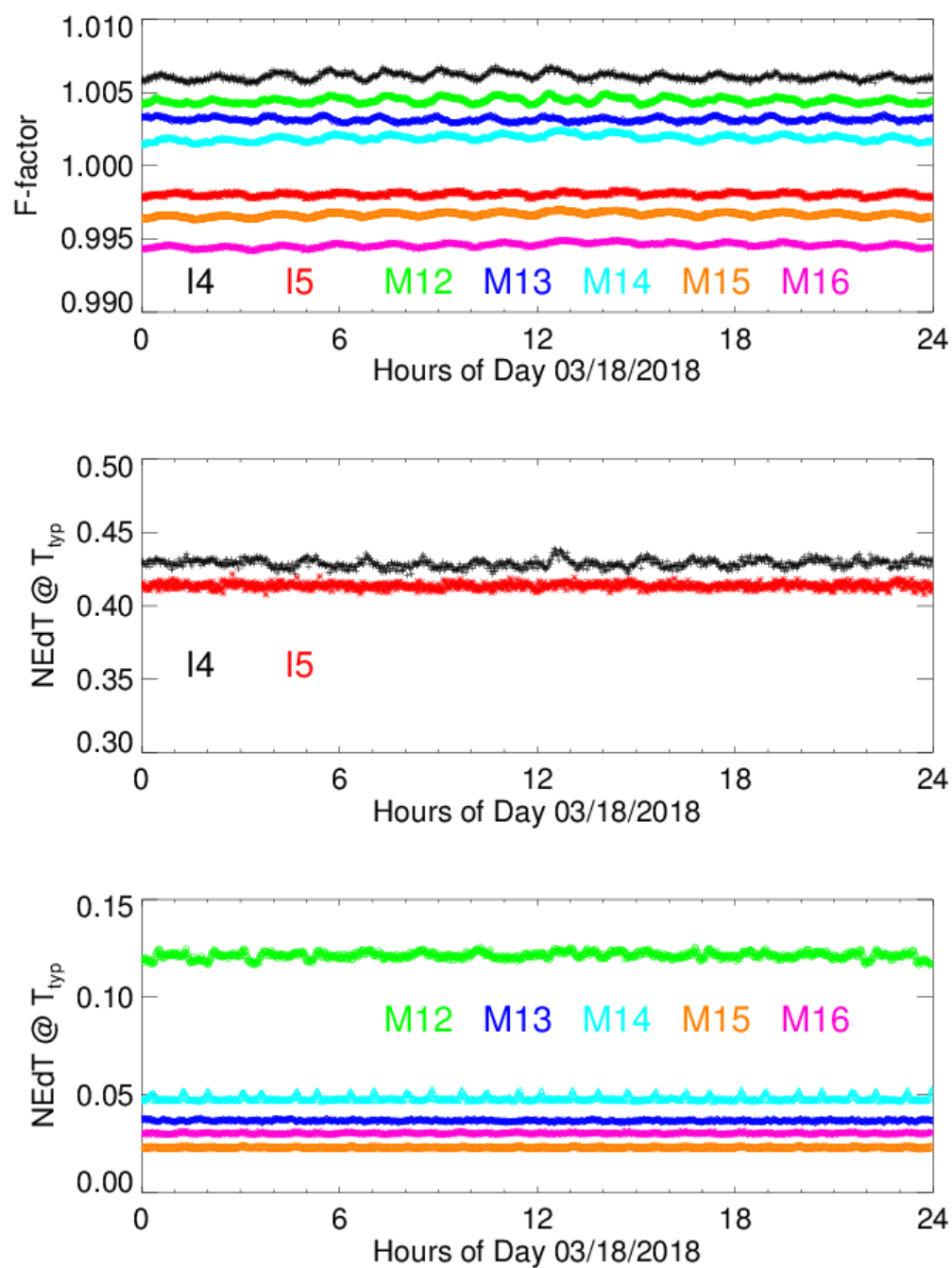

Figure 2. Daily trending of the granule and band-averaged F-factor and $N E d T_{\text {Ttyp }}$ on 03/18/2018.

Figure 2 shows the daily trending of the granule and band averaged F-factor and the NEdT at $\mathrm{T}_{\text {typ }}\left(\mathrm{NEdT}_{\text {Ttyp }}\right)$ for the TEBs. Slight orbital changes are seen in the F-factor trending. For a better visualization, the NEdT trending plots are grouped separately for the M- and I-bands. Larger NEdT $\mathrm{T}_{\text {Typ }}(0.4 \sim 0.45)$ is seen in the I-bands. The $\mathrm{NEdT}_{\text {Typ }}$ of all Mbands are below 0.1, except band M12. The NEdT of band M14 shows two little spikes per orbit which are not seen in any SNPP VIIRS TEB. The spikes of lower magnitude are also observed in band M16A.The spikes are not related to the geo-location of the granules. More investigations related to this issue will be performed in the future.

\subsection{N20 VIIRS TEB long-term trending}

For N20 VIIRS TEB, granule-averaged (48 scans) parameters are tracked in the long-term trending. One nighttime granule (near the South Pole) per day was selected and granules with invalid data caused by some instrument operation events were excluded. The locations of the selected granules (center coordinates of the granules) in the long-term trending analysis in this section are illustrated in Figure 3. 


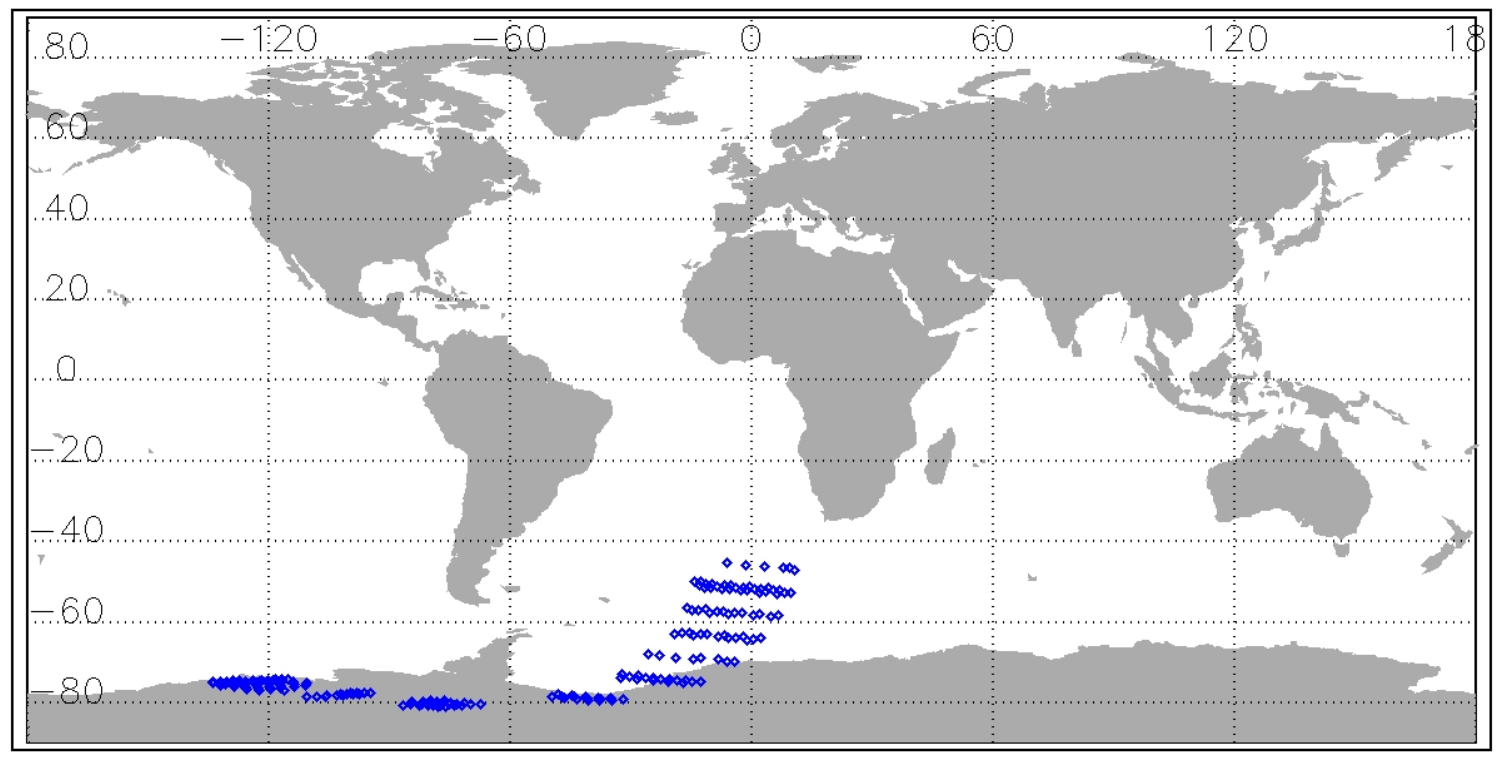

Figure 3. Locations of the selected granules (blue dots) for the long-term trending.

The temperatures of the two CFPAs are very stable (80.47 K for LWIR CFPA and $80.23 \mathrm{~K}$ for SMIR CFPA, varying within a range of $\sim 1 \mathrm{mK}$ and $\sim 17 \mathrm{mK}$, respectively). The granule averaged $\mathrm{T}_{\mathrm{bb}}$ is maintained at $292.6 \mathrm{~K}$ (during days 90 111 since launch it was raised to $307.5 \mathrm{~K}$ ) with about $5 \mathrm{mK}$ variation over the time when the BB is controlled at its nominal temperature, which means the BB provides a stable radiance reference for the TEB calibration. The long-term time series of the temperatures of the HAM, the cavity, the RTA, the shield, the OMM, and the electronics are illustrated in Figure 4. All of the temperatures were elevated when $T_{b b}$ was raised, except the electronics temperatures. The thermal radiation from the blackbody heats other components to a small degree.

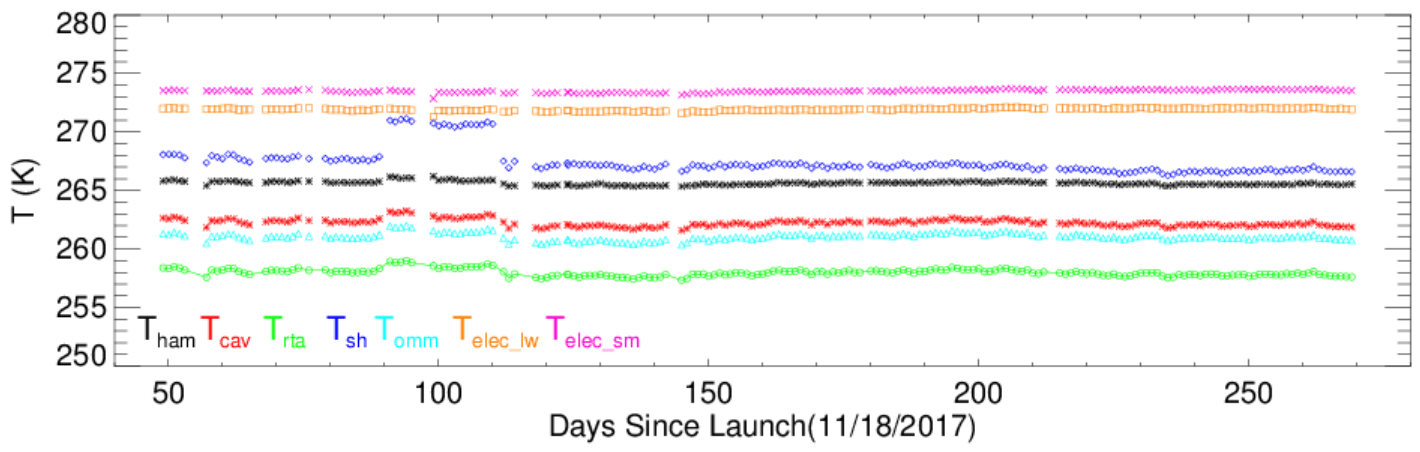

Figure 4. Long-term temperature trending.

Figure 5 shows the time series of the normalized band averaged F-factor. Upward trends in the early mission were seen in the LWIR bands I5, M14-16. This was believed to be caused by ice buildup in the optical path. The amount of drift in band M16 was the largest ( 1.5\%), followed by band I5 (1.4\%). The drift observed in band M14 was the smallest (0.1\%). The SMIR bands were not impacted. The F-factors of all TEBs are stable after the mid-mission outgas performed on March 12, 2018 (day 114 since launch). The F-factors for some detectors from their trending showed out-of-family behavior (e.g. I5d2, M12d16, M14d1\&d3, and M15d14), two detectors showed noisy trends (M13d2\&d16), two detectors in band M14 showed some gain changes (d6\&d14), etc. Since the N20 has only operated less than 300 days, 
more data needs to be collected to further understand the slight drift seen in the F-factor time series observed particularly in I5. Figure 6 shows the long-term trending of the $\mathrm{NEdT}_{\text {Ttyp. }}$. Except the period when the NEdT of all bands increased corresponding to the increase in $\mathrm{T}_{\mathrm{bb}}$ to $307.5 \mathrm{~K}$, the trends have been very stable since launch.

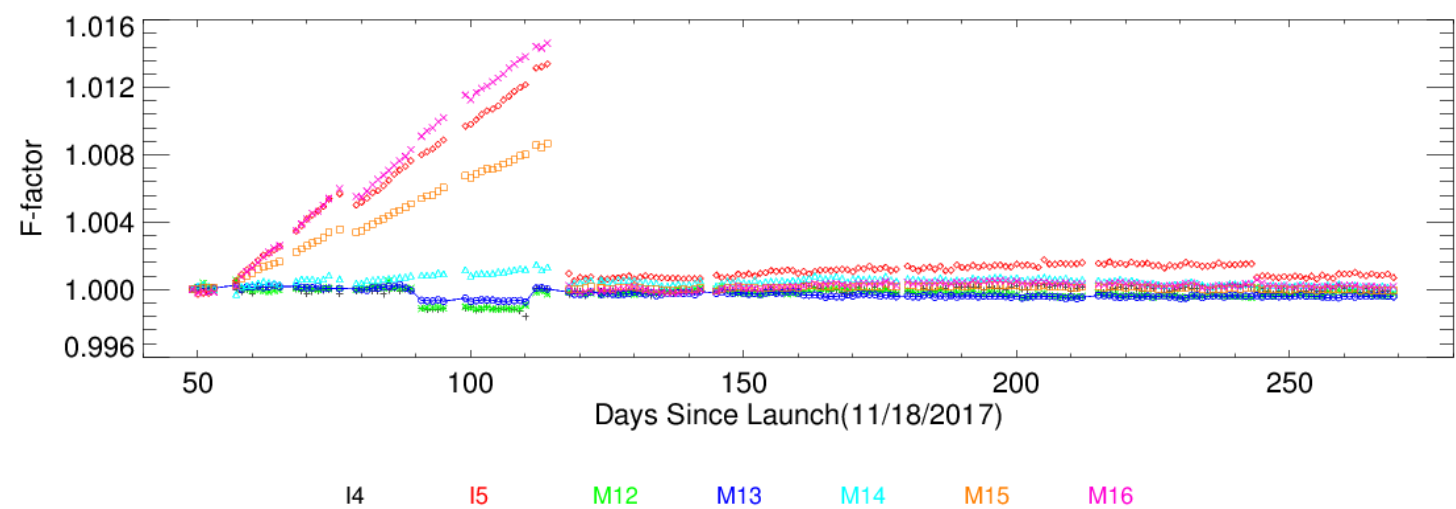

Figure 5. Long-term trending of the F-factors.

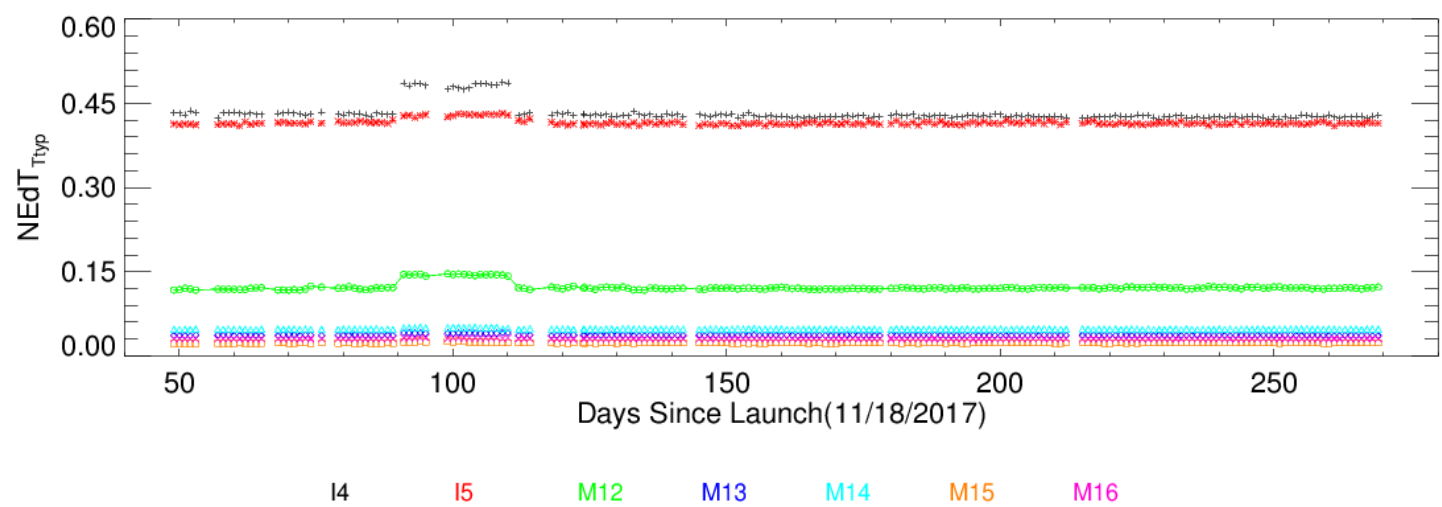

Figure 6. Long-term trending of the NEdT at $\mathrm{T}_{\text {typ. }}$.

\section{NOAA-20 VIIRS BB WUCD DATA ANALYSIS}

To monitor the on-orbit change of the TEB calibration coefficients, the VIIRS instrument operates the BB WUCD cycle to get the detector responses for the $\mathrm{T}_{\mathrm{bb}}$ in the range from $\sim 266 \mathrm{~K}$ to $315 \mathrm{~K}$. Since launch, the N20 VIIRS has performed four WUCD events. The first one was a full WUCD cycle operated on January 10, 2018. The BB was cooled down to its ambient temperature ( $266 \mathrm{~K}$ ) before the $\mathrm{WU}$ period. Then the $\mathrm{T}_{\mathrm{bb}}$ was set at 272.5, 282.5, 292.5, 297.5, 302.5, 307.5, 312.5 , and $315 \mathrm{~K}$ during the $\mathrm{WU}$ period. After the $\mathrm{T}_{\mathrm{bb}}$ reached to $315 \mathrm{~K}$, the $\mathrm{BB}$ heater was turned off and the $\mathrm{T}_{\mathrm{bb}}$ decreased to its ambient temperature. Then the BB was warmed up to its operational temperature through 272.5, 282.5, and $292.5 \mathrm{~K}$. The other three were short WUCD cycles, scheduled on January 22, April 9, and June 18, 2018. In the short WUCD cycle, the BB was heated directly from the operational temperature (292.6 K) and the $\mathrm{T}_{\mathrm{bb}}$ was set at 297.5, 302.5, 307.5, 312.5, and $315 \mathrm{~K}$ during the WU period. In the following calibration coefficient estimates, the results from both WU and CD granules are presented. 


\subsection{Calibration coefficients}

The calibration coefficients are estimated by quadratic fit of $\mathrm{dn}_{\mathrm{BB}}$ and the corresponding radiance $\mathrm{L}_{\text {det_ } \mathrm{BB}}$,

$$
L_{\text {det_BB }}=\sum_{i=0}^{2} C_{i} \cdot d n_{B B}^{i}
$$

The estimated calibration coefficients are actually the combination of the F-factor and the coefficients $C_{\mathrm{i}}$ in Eq. (1), which are now denoted by $C_{\mathrm{i}}(\mathrm{i}=0,1,2)$.

Figures 7-8 show the estimated band-averaged calibration coefficients from the four WUCD data for the SMIR bands and the LWIR bands, respectively. The results using the granules from both the WU (red) and the CD (blue) period are presented. The error bar is the standard deviation of the band averaged results, which represents the consistency of the detectors in a band. The green line is the coefficient from the prelaunch measurements. As seen in the plots, the coefficients of $\mathrm{C}_{0}$ and $\mathrm{C}_{2}$ extracted from the CD granules are more consistent across the four WUCD measurements. Slight variations in the first two points in the LWIR bands are likely due to the impact from the ice accumulation issue. More fluctuations among the detectors are seen in the results estimated from the WU granules. For the $\mathrm{C}_{1}$ coefficients, the error bars are not shown in the plots because the $\mathrm{C}_{1}$ values are related to the locations of the detector layout on the focal plane. In the case of LWIR bands, the estimated coefficients from CD granules are much closer to prelaunch values in comparison to those from the WU. Overall, the results from CD granules show better detector consistency than that from the WU granules, which is similar to the MODIS and the SNPP VIIRS ${ }^{3,4}$.

14

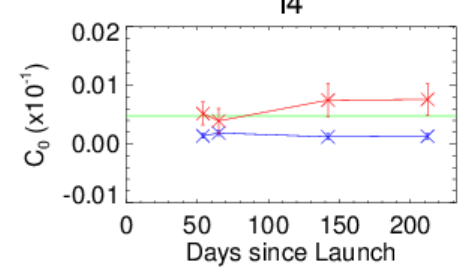

M12

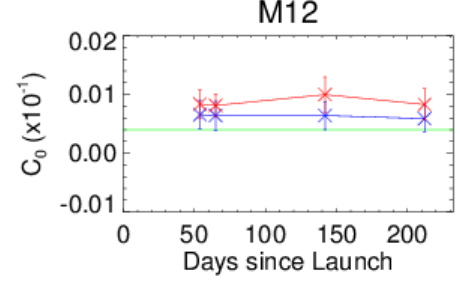

M13

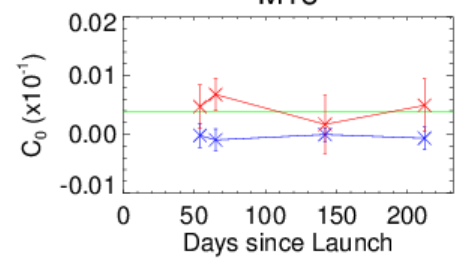

14

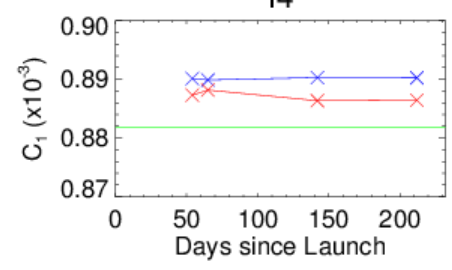

M12

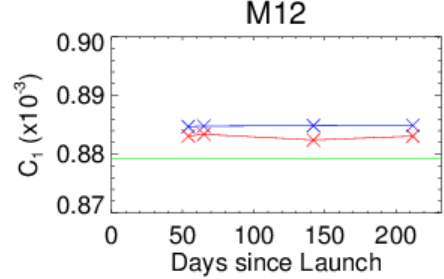

M13

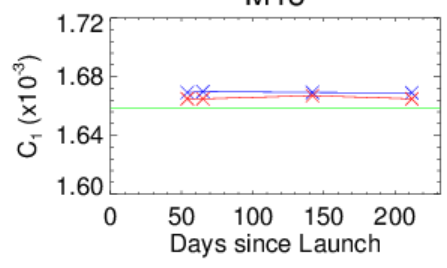

Prelaunch CD WU
14

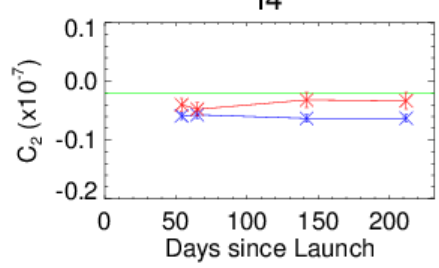

M12

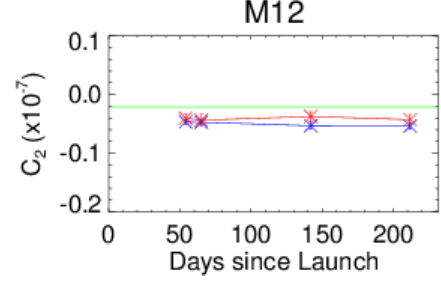

M13

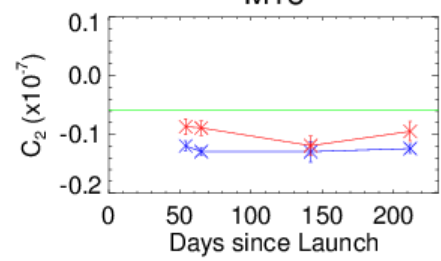

Figure 7. Band-averaged $C_{i}(i=0,1,2)$ coefficients derived from the BB WUCD data for the SMIR bands. 
15

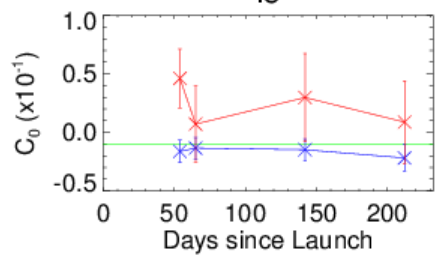

M14

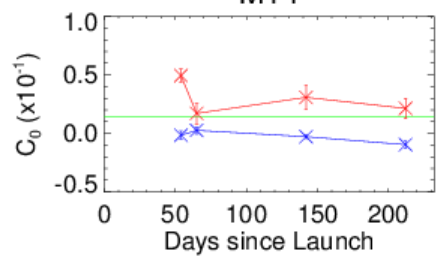

M15

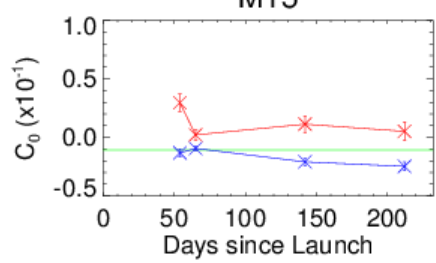

M16

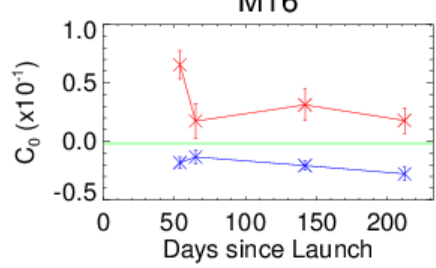

15

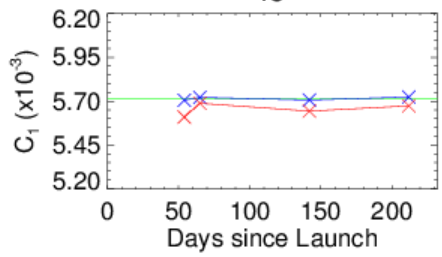

M14

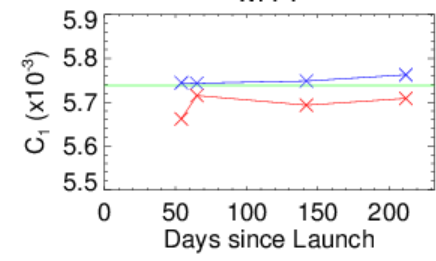

M15
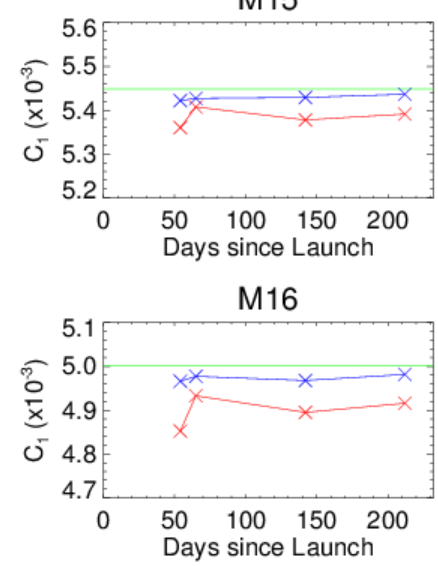

15

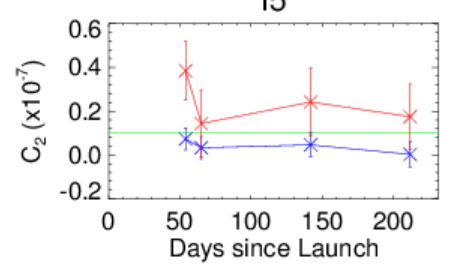

M14

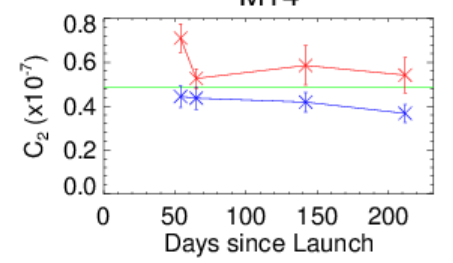

M15

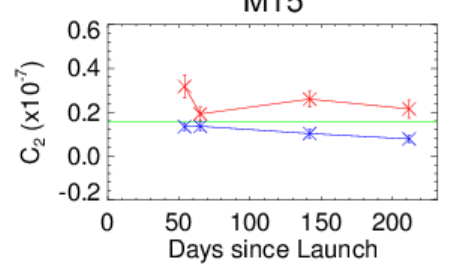

M16

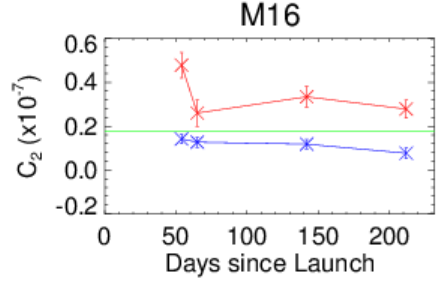

Prelaunch CD WU

Figure 8. Bands-averaged $C_{i}(i=0,1,2)$ coefficients derived from the BB WUCD data for the LWIR bands.

\subsection{Noise characterization at typical temperature}

The $\mathrm{T}_{\text {typ }}$ of most TEBs are covered in the WUCD cycle (see Table 1). This means that the $\mathrm{NEdT}_{\text {Ttyp }}$ can be obtained directly from the WUCD data. For band I5, its $\mathrm{T}_{\text {typ }}$ is lower than the lowest $\mathrm{T}_{\mathrm{bb}}(\sim 266 \mathrm{~K})$, but its $\mathrm{NEdT}_{\mathrm{Ttyp}}$ can still be estimated by fitting the data between $266 \mathrm{~K}$ and $315 \mathrm{~K}$.

Table 2 The NEdT at $\mathrm{T}_{\text {typ }}$ of the N20 VIIRS TEBs (unit: K).

\begin{tabular}{|c|c|c|c|c|c|c|c|c|c|c|}
\hline & I4S0 & I4S1 & I5S0 & I5S1 & M12 & M13 & M14 & M15 & M16 \\
\hline Prelaunch & \multicolumn{9}{|c|}{0.420} & \multicolumn{2}{c|}{0.410} & 0.120 & 0.043 & 0.050 & 0.026 & 0.043 \\
\hline \multicolumn{8}{|c|}{ WUCD } \\
\hline $\mathbf{2 0 1 8 0 1 1 1}$ & 0.381 & 0.381 & 0.338 & 0.307 & 0.099 & 0.037 & 0.047 & 0.023 & 0.031 \\
\hline $\mathbf{2 0 1 8 0 1 2 2}$ & 0.382 & 0.382 & 0.336 & 0.310 & 0.099 & 0.037 & 0.047 & 0.023 & 0.031 \\
\hline $\mathbf{2 0 1 8 0 4 0 9}$ & 0.383 & 0.383 & 0.338 & 0.307 & 0.100 & 0.037 & 0.047 & 0.023 & 0.031 \\
\hline $\mathbf{2 0 1 8 0 6 1 8}$ & 0.385 & 0.382 & 0.340 & 0.311 & 0.100 & 0.037 & 0.047 & 0.023 & 0.031 \\
\hline
\end{tabular}

Table 2 lists the values of the NEdT Ttyp calculated from all four on-orbit BB CD cycles. The NEdT values of a given band from the four WUCD cycles show excellent agreement. They are less than the prelaunch values ${ }^{5}$ and well below 


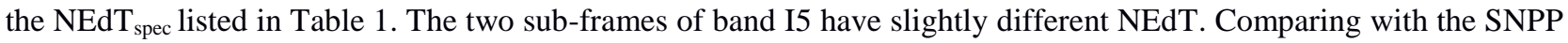
VIIRS, the N20 VIIRS bands I5, M12, M14, and M15 have slightly smaller NEdT ${ }_{\text {Ttyp }}$ while the other bands have nearly the same values ${ }^{4,6}$.

\section{SUMMARY}

Based on the all data in the N20 mission so far, all telemetry temperatures of instrument components that affect the TEB calibration and performance are stable. The F-factors (equivalent to the detector gains) appear stable for bands in the short- and mid-wave infrared CFPA. Some drift (up to 1.5\%) was seen in the LWIR bands in early mission likely because of ice buildup. After the mid-mission outgassing, the F-factors of all TEBs are stable. The calibration coefficients estimated from the four on-orbit BB cool-down data sets during the BB WUCD events are consistent and in reasonable agreement with the prelaunch values. The NEdT at typical temperature of each band is well below its designed specification and close to that of the SNPP VIIRS. Even though some detectors in bands I5, M12-15 behave slightly noisy or out-of-family in their F-factors, the N20 VIIRS is performing very well. The cause of the spikes seen in band M14 NEdT trending will be further investigated.

\section{REFERENCES}

[1] Joint Polar Satellite System (JPSS). "VIIRS Radiometric Calibration Algorithm Theoretical Basis document (ATBD), Revision C,” Goddard Space Flight Center, Greenbelt, MD, USA. [Online] Available online: https://www.star.nesdis.noaa.gov/jpss/documents/ATBD/D0001-M01-S01-003_JPSS_ATBD_VIIRS-SDR_D.pdf (2013).

[2] McIntire, J., Moyer, D., Oudrari, H., and Xiong, X., "Pre-Launch Radiometric Characterization of JPSS-1 VIIRS Thermal Emissive Bands,” Remote Sens., 8(1), 47 (2016).

[3] Wenny, B.N., Wu, A., Madhavan, S., Wang, Z., Li, Y., Chen, N., Chiang, K., and Xiong, X., "MODIS TEB calibration approach in collection 6,” Proc. SPIE 8533, 85331M (2012).

[4] Efremova, B., McIntire, J., Moyer, D., Wu, A., and Xiong, X., "S-NPP VIIRS thermal emissive bands on-orbit calibration and performance,” J. Geophys. Res. Atmos., 119, 10,859-10,875 (2014).

[5] Oudrari, H., McIntire, J., Xiong, X., Butler, J., Ji, Q., Schwarting, T., Lee, S., and Efremova, B., “JPSS-1 VIIRS Radiometric Characterization and Calibration Based on Pre-Launch Testing,” Remote Sens., 8, 41 (2016).

[6] Xiong, X., Butler, J., Chiang, K., Efremova, B., Fulbright, J., Lei, N., McIntire, J., Oudrari, H., Sun, J., Wang, Z., and Wu, A., "VIIRS on-orbit calibration methodology and performance," J. Geophys. Res. Atmos., 119, 1-11 (2013). 